\title{
ORIGINAL ARTICLE Strong seduction: impulsivity and the impact of contextual cues on instrumental behavior in alcohol dependence
}

\author{
C Sommer $^{1}$, M Garbusow ${ }^{2}$, E Jünger ${ }^{1}$, S Pooseh ${ }^{1}$, N Bernhardt ${ }^{1}$, J Birkenstock ${ }^{1}$, DJ Schad ${ }^{3}$, B Jabs ${ }^{4}$, T Glöckler ${ }^{4}$, QM Huys $^{5,6}$, A Heinz ${ }^{2}$, \\ MN Smolka ${ }^{1}$ and US Zimmermann ${ }^{1}$
}

\begin{abstract}
Alcohol-related cues acquire incentive salience through Pavlovian conditioning and then can markedly affect instrumental behavior of alcohol-dependent patients to promote relapse. However, it is unclear whether similar effects occur with alcohol-unrelated cues. We tested 116 early-abstinent alcohol-dependent patients and 91 healthy controls who completed a delay discounting task to assess choice impulsivity, and a Pavlovian-to-instrumental transfer (PIT) paradigm employing both alcohol-unrelated and alcoholrelated stimuli. To modify instrumental choice behavior, we tiled the background of the computer screen either with conditioned stimuli (CS) previously generated by pairing abstract pictures with pictures indicating monetary gains or losses, or with pictures displaying alcohol or water beverages. CS paired to money gains and losses affected instrumental choices differently. This PIT effect was significantly more pronounced in patients compared to controls, and the group difference was mainly driven by highly impulsive patients. The PIT effect was particularly strong in trials in which the instrumental stimulus required inhibition of instrumental response behavior and the background CS was associated to monetary gains. Under that condition, patients performed inappropriate approach behavior, contrary to their previously formed behavioral intention. Surprisingly, the effect of alcohol and water pictures as background stimuli resembled that of aversive and appetitive CS, respectively. These findings suggest that positively valenced background CS can provoke dysfunctional instrumental approach behavior in impulsive alcohol-dependent patients. Consequently, in real life they might be easily seduced by environmental cues to engage in actions thwarting their longterm goals. Such behaviors may include, but are not limited to, approaching alcohol.
\end{abstract}

Translational Psychiatry (2017) 7, e1183; doi:10.1038/tp.2017.158; published online 1 August 2017

\section{INTRODUCTION}

Environmental cues are important moderators of ongoing instrumental behavior. Via Pavlovian learning, previously neutral cues can acquire motivational value by predicting the occurrence of rewarding or punishing events. In substance dependence, stimuli previously associated with drug reward enhance craving, ${ }^{1}$ promote approach, ${ }^{2}$ and may thus lead to relapse. ${ }^{3}$

Experimental approaches to study the influence of Pavlovian conditioned cues on instrumental responses are Pavlovian-toinstrumental transfer (PIT) paradigms (for example, Holmes et al. ${ }^{4}$ ). They combine instrumental learning, where a reward enhances or decreases the occurrence of a specific action, with Pavlovian, where learning confers incentive salience to previously neutral cues by pairing them with a reward. The interaction between Pavlovian cues and instrumental behavior can be specific, that is, when a Pavlovian cue promotes instrumental behavior specifically associated with the same reward. In contrast, general PIT denotes that, depending on the motivational valence, Pavlovian cues can moderate instrumental approach and avoidance responses in general independent of reward types. ${ }^{5,6}$ Thereby, positively valued Pavlovian cues encourage approach tendencies, while negative cues promote inhibition and avoidance actions. ${ }^{7}$ PIT is considered to be an essential mechanism in behaviors that are subject to cuecontrol, such as eating, alcohol use and drug-taking.
PIT paradigms have been extensively used in animal studies. ${ }^{4}$ Regarding substance dependence, stronger specific PIT effects were reported in rats after repeated cocaine or amphetamine administration, compared to drug-naive animals. ${ }^{8,9}$ Notably, pretreatment with cocaine also enhanced general PIT, where neither Pavlovian cues nor instrumental responses were drugrelated. ${ }^{10}$

Related to that, studies in humans demonstrated attentional biases towards non-drug related (for example, monetary) rewards leading to impaired goal-directed inhibition. ${ }^{11}$ Interestingly, those attentional biases were found to be greater in opioid-dependent patients compared to controls. ${ }^{12}$ Adapting animal PIT paradigms in humans confirmed that Pavlovian cues can influence instrumental responding in healthy human subjects. ${ }^{7,13-15}$ In the context of alcohol dependence, our group recently reported that PIT was more pronounced in alcohol-dependent patients compared to controls. ${ }^{16}$ In a larger sample, we also observed that the PIT effect of non-drug-related cues was positively related to stronger signals in the nucleus accumbens (NAcc) only in relapsers, but not in abstainers. ${ }^{17}$ These findings demonstrate that the degree to which Pavlovian cues affect behavior varies markedly across subjects, which might be further influenced by personality traits.

\footnotetext{
${ }^{1}$ Department of Psychiatry and Psychotherapy, Technische Universität Dresden, Dresden, Germany; ${ }^{2}$ Department of Psychiatry and Psychotherapy, Charité-Universitätsmedizin Berlin, Campus Charité Mitte, Berlin, Germany; ${ }^{3}$ Social and Preventive Medicine, Area of Excellence Cognitive Sciences, University of Potsdam, Potsdam, Germany; ${ }^{4}$ Department of Psychiatry and Psychotherapy, Zentrum für Psychische Gesundheit Weißer Hirsch, Dresden, Germany; ${ }^{5}$ Translational Neuromodeling Unit, Hospital of Psychiatry, University of Zürich and Swiss Federal Institute of Technology, Zürich, Switzerland and ${ }^{6}$ Psychiatry, Psychosomatics and Psychotherapy, University of Zürich, Zürich, Switzerland. Correspondence: Professor US Zimmermann, Department of Psychiatry and Psychotherapy, University Hospital Carl Gustav Carus, Fetscherstraße 74, Dresden 01307, Germany. E-mail: Ulrich.Zimmermann@uniklinikum-dresden.de

Received 12 May 2017; accepted 13 June 2017
} 
One important personality trait that is known to be a risk factor for initiating drug and alcohol use as well as addiction is impulsivity. ${ }^{18}$ Impulsivity is a multifaceted construct that has been operationalized in many ways. ${ }^{19-21}$ One of its components is the sensitivity of choices to delay, as assessed by delay discounting tasks, where impulsive choice behavior is defined as the tendency to prefer immediate over delayed rewards. There is a conceptual similarity between models of addiction and the tendency to prefer immediate over delayed rewards, since addictive behaviors are characterized by choosing to consume a substance in the present, often at the expense of concern about future outcomes. ${ }^{22}$ There are numerous studies reporting higher delay discounting in alcohol-dependent patients compared to controls (for a metaanalysis see Mackillop et $a l^{23}$ ), and within patients, higher discounting rates seem to be sensitive for the prediction of relapse. ${ }^{24}$ On the neural level, impulsive decisions are linked to stronger activation in limbic regions including amygdala and NAcc, ${ }^{25,26}$ which are also reported to play a key role during PIT (for example, ${ }^{27,28}$ ).

The current study pursued three aims. First, we wanted to replicate our previous finding that severely alcohol-dependent patients compared to controls show a stronger general PIT effect $^{16,17}$ by now analyzing both, a clean replication sample $(n=130)$ where we excluded subjects of previously reported subsamples and the full sample containing all assessed subjects $(n=207)$. Second, due to the bigger sample size, we can provide a more exhaustive analysis to explore whether positive and negative Pavlovian cues differ in their potency to separately influence approach and avoidance behavior, as noted by Geurts et al. ${ }^{29}$ Third, based on the described literature linking impulsive choice behavior to PIT, we tested whether more impulsive individuals exhibit stronger PIT effects compared to less impulsive subjects.

\section{MATERIALS AND METHODS}

All procedures complied with the Declaration of Helsinki and were approved by the ethical committees of the Charité-Universitätsmedizin Berlin and of the the Technische Universität Dresden.

\section{Participants}

Data were collected in Berlin and Dresden as part of the LeAD study (Learning and Alcohol Dependence; www.leadstudie.de; clinical trial number: NCT01679145). We included 18-65-year-old male and female alcohol-dependent patients who were abstinent from alcohol by a mean of 17 days (s.d. $=11$ ) as well as age- and gender-matched healthy controls. Exclusion criteria for all subjects were left-handedness, MRcontraindications (for example, metal-containing foreign body, pacemaker, and claustrophobia), a history of dependence or current substance use except for nicotine; other current DSM-IV axis one psychiatric or neurologic disorders and borderline personality disorder. Included patients were severely alcohol-dependent with a history of alcohol dependence for a minimum of 3 years and had been recently detoxified. Patients were off any medication known to interact with the central nervous system (more than four half-lives post last intake) and displayed no relevant alcohol withdrawal symptoms (CIWA-Ar score $\geqslant 3$ ). ${ }^{30}$ We assessed 212 participants fulfilling all criteria. Due to technical problems in either the delay discounting or the PIT task, data of 5 participants were missing, resulting in a final sample of 116 patients and 91 age- and gender-matched healthy controls. For sample characteristics, see Table 1.

\section{General experimental procedures}

Participants underwent two experimental days. On the first day, after giving written informed consent, participants were interviewed using the computerized Composite International Diagnostic Instrument ${ }^{37,38}$ to check for DSM-IV axis I disorders. Following the interview, participants filled out several self-rating questionnaires (Table 1) before they completed a delay discounting task. On the second day, participants completed a PIT paradigm. We excluded current alcohol use by breath alcohol readings at the beginning of both experimental days. At the end of each day,

\begin{tabular}{|c|c|c|c|c|c|}
\hline \multirow[b]{2}{*}{$N$} & \multicolumn{2}{|c|}{ Patients } & \multicolumn{2}{|c|}{$\begin{array}{l}\text { Healthy } \\
\text { controls }\end{array}$} & \multirow{2}{*}{$\frac{\mathrm{t} \text {-test } / X^{2}}{0.85^{\mathrm{a}}}$} \\
\hline & \multicolumn{2}{|c|}{$\begin{array}{l}116 \text { (16 } \\
\text { females) }\end{array}$} & \multicolumn{2}{|c|}{$\begin{array}{c}91 \text { (14 } \\
\text { females) }\end{array}$} & \\
\hline \multirow[t]{2}{*}{ \% Smokers } & \multicolumn{2}{|c|}{$78.4 \%$} & \multicolumn{2}{|c|}{$68.1 \%$} & $0.13^{\mathrm{a}}$ \\
\hline & Mean & s.d. & Mean & s.d. & $P$ \\
\hline Age in years & 44.88 & 10.43 & 43.50 & 11.12 & 0.37 \\
\hline Verbal intelligence score & 104.50 & 9.47 & 103.94 & 8.91 & 0.66 \\
\hline Digit span backwards & 4.79 & 1.12 & 5.24 & 1.14 & $<0.01$ \\
\hline Socioeconomic status score & -0.49 & 1.93 & 0.65 & 2.04 & $<0.001$ \\
\hline Education (years) & 0.15 & 0.97 & 0.20 & 1.01 & $<0.05$ \\
\hline HADS anxiety & 4.56 & 3.40 & 2.37 & 2.05 & $<0.001$ \\
\hline HADS depression & 3.82 & 3.78 & 1.83 & 2.28 & $<0.001$ \\
\hline Delay discounting $(k)$ & -3.22 & 3.56 & -4.41 & 3.01 & $<0.01$ \\
\hline $\begin{array}{l}\text { Time since last alcohol } \\
\text { consumption }\end{array}$ & 17.23 & 10.86 & 22.98 & 57.54 & 0.46 \\
\hline Lifetime alcohol intake (kg) & 1118 & 1131 & 301 & 840 & $<0.001$ \\
\hline $\begin{array}{l}\text { Alcohol intake per day past } \\
\text { year }(\mathrm{g})\end{array}$ & 179 & 138 & 11 & 14 & $<0.001$ \\
\hline Alcohol dependence scale & 14.77 & 6.94 & 2.19 & 3.11 & $<0.001$ \\
\hline OCDS total score & 11.89 & 8.54 & 2.66 & 2.83 & $<0.001$ \\
\hline
\end{tabular}

Abbreviations: ADS, alcohol dependence scale ${ }^{31}$ delay discounting $(k)$, logarithmic scale of $k$ extracted from the delay discounting task; digit span backwards (verbal working memory capacity, WAIS-II), ${ }^{32}$ individual number of correctly repeated digits in reverse order; education years, $z$-transformed years in school, university and vocational training; HADS, Hospital Anxiety and Depression Scale; OCDS, Obsessive Compulsive Drinking Scale, German version; ${ }^{33}$ SES, socioeconomic status; verbal intelligence score, $z$-transformed scores of the Mehrfachwahl-Wortschatz-Intelligenztest (MWT-B). ${ }^{34}$ a $P$-value of $X^{2}$-test. SES is computed as the sum of $z$-transformed social status, household income and inverse personal debt scores. ${ }^{35}$ HADS scores from 0 to 7 are defined as not clinically relevant. ${ }^{36}$

participants received task winnings and a monetary compensation for study participation, resulting in an average payout of $10 € /$ hour.

\section{Delay discounting task}

Participants completed an adaptive delay discounting task which is part of a value-based decision-making task-battery. ${ }^{39}$ On each of 30 trials, subjects chose between a small immediate and a larger delayed monetary reward. Delays were set to $3,7,14,31,61,180$, or 365 days. Rewards varied between $0.30 €-10 €$. Participants were informed that one of their choices was credited to their compensation at the end of the experiment. On the basis of the choices, we estimated individual delay discounting rates $k$ assuming a hyperbolic discounting function as proposed by Mazur ${ }^{40}$ and a softmax decision function. Because the distribution of $k$ was skewed, statistical analyses were performed on the natural logarithmic scale.

\section{PIT paradigm}

On the second experimental day, participants completed a PIT paradigm (Figure 1). ${ }^{7,29}$ The task was programmed using Matlab 2011 (MATLAB version 7.12.0, 2011; MathWorks, Natick, MA, USA) with the Psychophysics Toolbox Version 3 (PTB-3) extension. ${ }^{41,42}$ The PIT task consisted of four sequential parts: (1) instrumental training, (2) Pavlovian training, (3) PIT and (4) a forced choice task. The instrumental training was conducted before and the forced choice task after the functional-MRI (fMRI), while the Pavlovian training and PIT part were assessed during fMRI scanning.

\section{Instrumental training}

Subjects were instructed to collect 'good' shells and leave 'bad' shells by repeatedly pressing a button while receiving probabilistic feedback. 


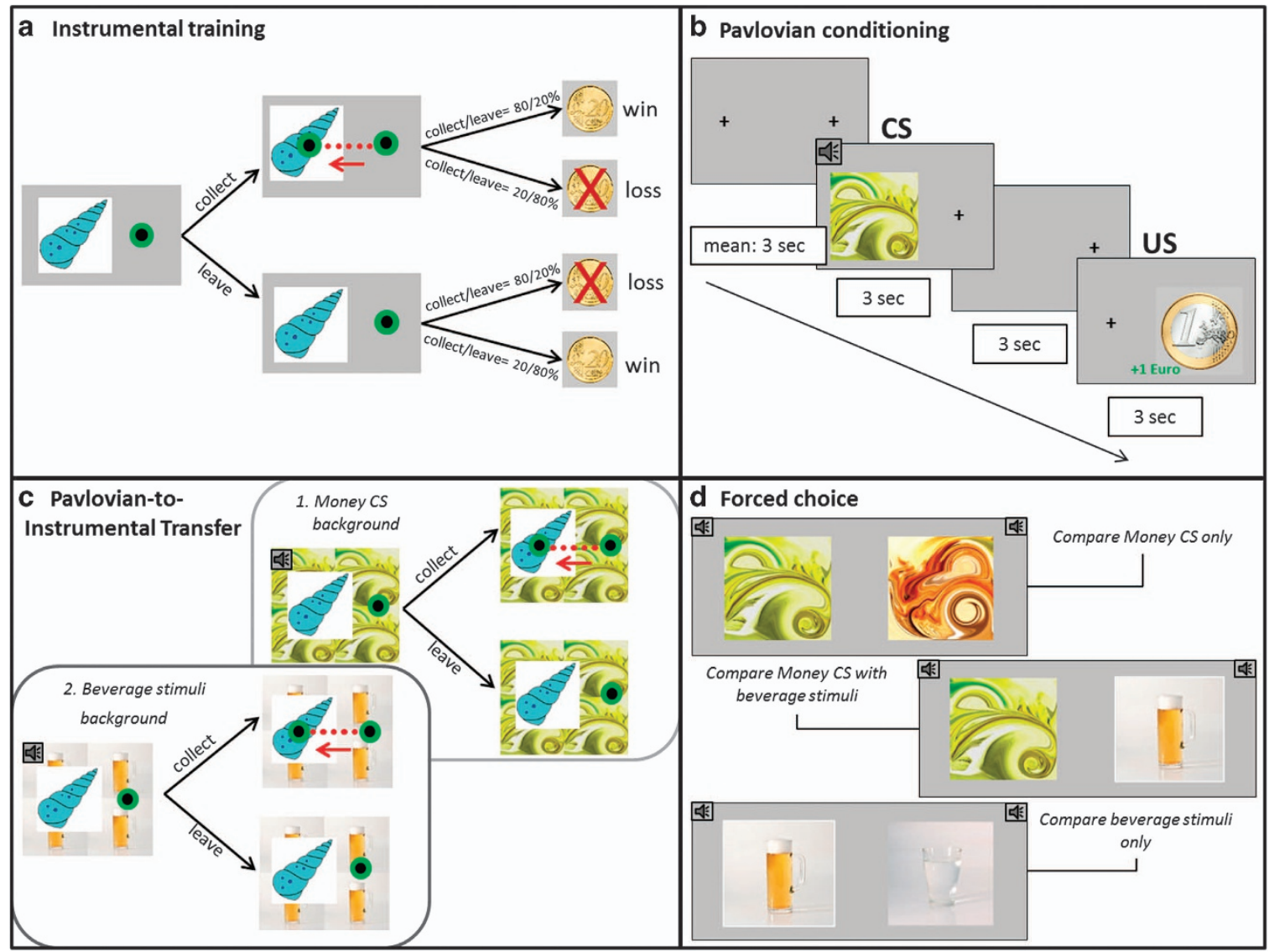

Figure 1. Pavlovian-to-instrumental transfer (PIT) task consisted of four parts: (a) Instrumental training: To collect a 'good' shell, subjects had to move a red dot onto the shell by repeatetly pressing a button. To leave a 'bad' shell, subjects were instructed to do nothing. Correct responses were rewarded with 20 Cents in $80 \%$ of the trials and punished by loosing 20 Cents in $20 \%$ of trials, and vice versa for incorrect responses. Participants performed between 60 and 120 trials. (b) Pavlovian Training: Audio-visual compound cues (CS) were deterministically associated with one of five outcomes $(-2 €,-1 €, 0 €, 1 €, 2 €)$. Participants performed 80 trials. (c) PIT: Subjects performed the instrumental task again, this time without outcome feedback. The background was tiled with one of the money CS presented during Pavlovian training, or one of 4 beverage stimuli. Participants performed 162 trials. (d) Forced-choice trials: (i) subjects were faced with a choice between two money CS (30 trials); (ii) or a money CS and a beverage stimulus (alcohol or water, 60 trials), (iii) between two beverage stimuli (18 trials).

Participants had to press the button 5 or more times in order to collect a good shell (Collect-trials) and do nothing (perform 0-4 button presses) to leave a bad shell (Leave-trials). Correct responses were rewarded with 20 Cents in $80 \%$ of the trials and punished with a loss of 20 Cents in $20 \%$ of trials, and for wrong responses it was vice versa (Figure 1a). We presented six different shells while each trial lasted $2 \mathrm{~s}$. Participants performed between 60 and 120 trials, depending on their performance: in an effort to ensure that all subjects were at comparable performance levels, a learning criterion stopped instrumental training if participants performed $80 \%$ correct choices over 16 consecutive trials.

\section{Pavlovian training}

For Pavlovian conditioning, 80 trials of pairing monetary rewards or punishments with compound visual and auditory CS were presented to the subjects. At the beginning of each trial, a compound CS consisting of abstract pictures and pure tones was presented for $3 \mathrm{~s}$. The compound CS was followed by a delay of $3 \mathrm{~s}$ with two fixation crosses at the two potential CS locations (left and right), then a US (monetary reward or punishment) was presented for a further $3 \mathrm{~s}$ (Figure 1b). CS and US presentation was separated with a 3 -second interstimulus interval. Subjects were instructed to passively observe the CS and US and to memorize the pairings. The set of stimulus pairings consisted of two positive CS paired with images of $+2 €$ and $+1 €$ coins, respectively; one neutral CS paired with $0 €$ and two negative CS paired with $-1 €$ and $-2 €$, respectively (coins with a superimposed red cross).
Pavlovian-to-instrumental transfer

Subjects performed the instrumental task again, this time without outcome feedback. The background was tiled with one of the money CS presented during Pavlovian training, or one of 4 beverage stimuli ( 2 pictures of the favorite alcoholic drink and 2 pictures of water). Participants performed 162 trials while each trial lasted $3 \mathrm{~s}$ (Figure 1c).

\section{Forced-choice task}

A forded choice task was performed in order to verify acquisition of Pavlovian expectations. For each trial, subjects had to choose one of two sequentially presented compound CS (Figure 1d). All possible compound CS pairings were presented three times in an interleaved, randomized order and stimuli were presented one at a time for $2 \mathrm{~s}$.

\section{Data analysis}

Data were analyzed using R 3.2.0 (R Development Core Team, Vienna, Austria, 2014). ${ }^{43}$ Demographic group characteristics and group comparisons were examined using chi-square and $t$-tests (Table 1). While all further described analyses refer to the transfer part of our experiment (Figure 1c), analyses of the instrumental training and the forced choice trails can be found in the supplement (Supplementary Figures 2 and 3).

In a first step, we replicated our previous finding that patients compared to controls show a stronger general PIT effect ${ }^{16,17}$ by creating a clean replication sample, excluding all subjects of those previously reported studies. To analyze differences in accuracy between patients and controls, 
we used binomial mixed-effects models (glmer, R-package: Ime4). We report Bonferroni-corrected estimates, standard errors, $Z$-values and $P$-values from the glmer output. We coded participants' response for each trial as correct $(=1)$ if a good shell was collected or a bad shell was left, and as false $(=0)$ if a bad shell was collected or a good shell was left, respectively. In a second step, we used the same models to analyze the transfer part within our full sample. Finally, to check for the influence of impulsivity on the PIT effect in the full sample, we extracted the parameter $k$ from the delay discounting task. Since the range of $k$ was approximately equal in patients ( $\operatorname{Min}=-13.3$; Max =4.3) and in controls (Min = 12.6; Max = 5.1), we performed a median split to divide the whole sample in high $k$ vs low $k$ subjects. After median split, the high $k$ group included 68 patients and 35 controls, and the low $k$ group included 48 patients and 56 controls.

\section{RESULTS}

Replication sample

Effect of money CS in the background ('money PIT'). To predict accuracy for trials with money CS backgrounds within the replication sample, we calculated a model including the fixed factors money $\mathrm{CS}(-2 €,-1 €, 0 €, 1 €, 2 €$, linear), type of
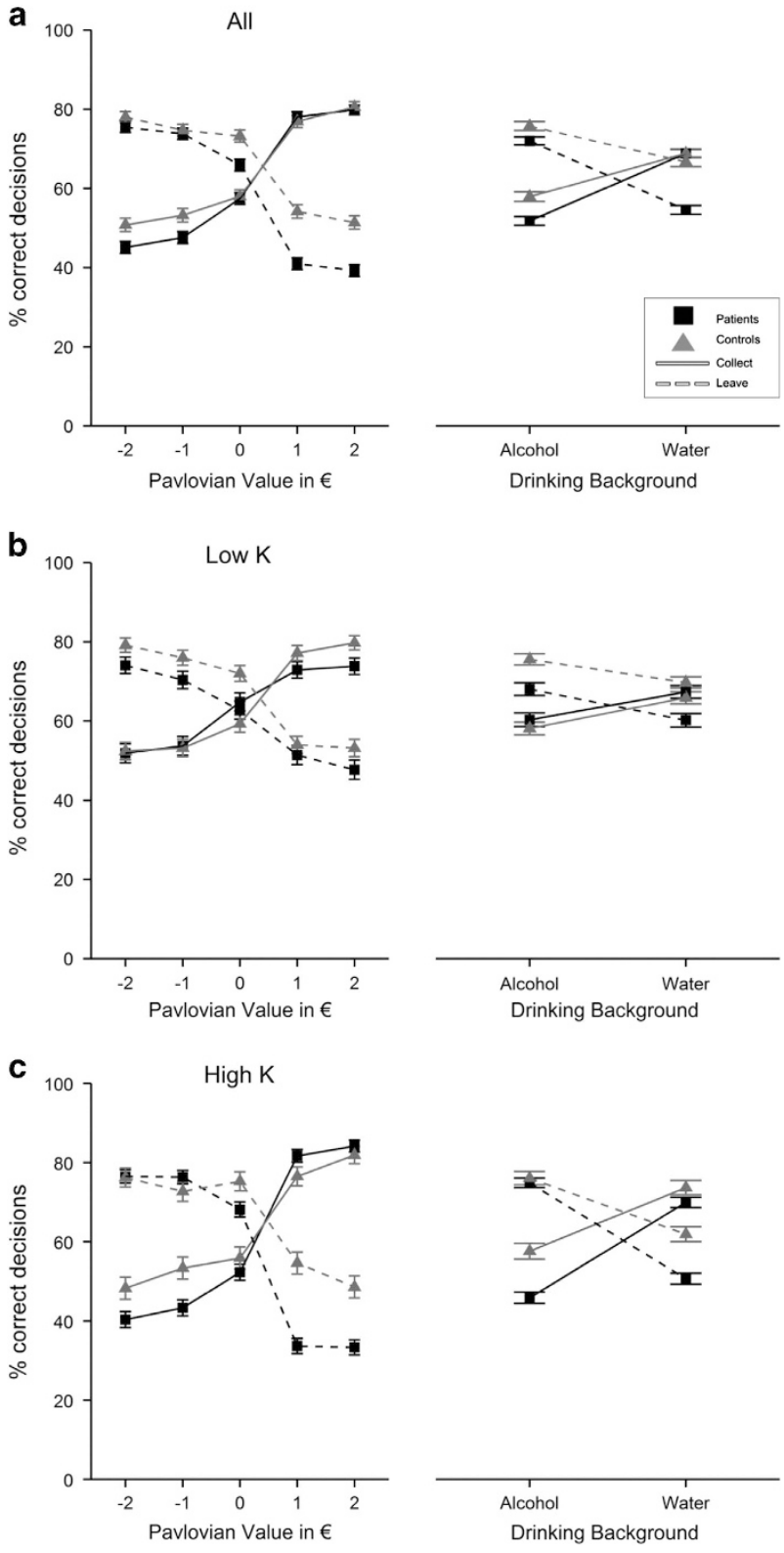

instrumental behavior $(0.5=$ collect good shell vs $-0.5=$ leave bad shell) and group $(0.5=$ patients vs $-0.5=$ controls $)$, as well as the random intercept. We found a stronger PIT effect in patients compared to controls for leaving bad shells (Estimate $=0.16, z=3.66$, $P<0.001$ ), suggesting that with an increasing value of background $\mathrm{CS}$, patients compared to controls were less able to accurately refrain from collecting bad shells (Supplementary Figure 1A).

Effect of beverage stimuli in the background ('beverage PIT'). To analyze trials with beverage backgrounds, we replaced the fixed factor money CS with the factor beverage $(0.5=$ alcohol vs $0.5=$ water $)$. Again, we found a stronger PIT effect in patients compared to controls, both for collecting good (Estimate $=-0.44, z=-2.93, P=0.003$ ) and leaving bad shells (Estimate $=0.51, z=3.65, P<0.001$ ), suggesting that patients compared to controls were less able to correctly collect good shells when alcohol pictures were presented in the background as well as they were less able to correctly leave a bad shell when water pictures were presented (Supplementary Figure 1B).

\section{Full sample}

Effect of money CS in the background ('money PIT'). To predict accuracy for trials with money CS backgrounds within the full sample, we calculated a model including the fixed factors money CS $(-2 €,-1 €, 0 €, 1 €, 2 €$, linear), type of instrumental behavior $(0.5=$ collect good shell vs $-0.5=$ leave bad shell) and group ( $0.5=$ patients vs $-0.5=$ controls), as well as the random intercept. We found a significant interaction of money CS $\times$ type of instrumental behavior (Estimate $=0.91, z=36.62, P<0.001$ ), indicating a PIT effect in both groups: when collecting good shells, subjects responded more often correctly with increasing value of money CS in the background, whereas for leaving bad shells they responded more often correctly with decreasing value of money $\mathrm{CS}$. The significant interaction money $\mathrm{CS} \times$ type of instrumental behavior $\times$ group (Estimate $=-0.18, z=-3.69, P<0.001$ ) revealed that patients and controls differed in the strength of PIT. When calculating separate models for collecting good shells and leaving bad shells, we found a significant interaction of money $\mathrm{CS} \times$ group only in the model for leaving bad shells (Estimate $=0.12, z=3.43$, $P<0.001$ ), suggesting that with an increasing value of background CS, patients compared to controls were less able to accurately leave bad shells (see Figure $2 \mathrm{a}$, left panel).

Modulation of money PIT by choice impulsivity. Including $k$ (high $k$ vs low $k$, indicating high vs low impulsive choice behavior) in

Figure 2. PIT effects are shown as changes of correct responses as a function of Pavlovian background value or drinking backgrounds, respectively. High and low $k$ groups were obtained by median split. (a) Left panel: Patients and controls showed PIT effects: when collecting good shells, participants accuracy increased with increasing value of background CS, while for leaving bad shells, accuracy increased with decreasing CS values. Patients had stronger PIT effects than controls when leaving bad shells. (a) Right panel: Participants collected good shells more correctely when water backgrounds were presented and left bad shells more correctly when alcohol backgrounds were presented. Patients showed a stronger PIT effect than controls when leaving bad shells. (b) Left panel: When collecting good shells, low $k$ controls responded more correctly with increasing value of background CS compared to low $k$ patients. (b) Right panel: We found no significant group differences when beverage backgrounds were presented. (c) Left panel: When confronted with bad shells, high $k$ patients responded less correctly than high $k$ controls with increasing value of background CS. (c) Right panel: When confronted with beverage backgrounds, high $k$ patients showed stronger PIT effects than high $k$ controls when leaving bad shells. CS, conditioned stimuli; PIT, Pavlovian-toinstrumental transfer. 
the above described model revealed a significant three-way interaction between money CS $\times$ type of instrumental behavior $\times k$ (Estimate $=0.31, z=6.19, P<0.001$ ), indicating that impulsive choice behavior modulated the PIT effect. Further, this modulation differed between patients and controls, as suggested by the significant four-way interaction between money $\mathrm{CS} \times$ type of instrumental behavior $\times k \times$ group (Estimate $=-0.59, z=-5.82$, $P<0.001)$. To disentangle this interaction, we computed separate models for collecting good and leaving bad shells for low $k$ and high $k$ participants, on one hand, and for patients and controls on the other. For low $k$ participants collecting good shells, a significant interaction of money $\mathrm{CS} \times$ group revealed that patients compared to controls were less affected by money CS (Estimate $=$ $-0.14, z=2.63, P<0.009$; see Figure $2 b$, left panel). For low $k$ participants leaving bad shells, we found no differences between patients and controls. We found no significant effects for high $k$ subjects collecting good shells, but when high $k$ subjects were confronted with bad shells, patients compared to controls were more affected by money CS (Estimate $=0.26, z=4.80, P<0.001$; see Figure $2 c$, left panel). In the model including only patients, we found significant interactions of money $\mathrm{CS} \times k$ indicating that high $k$ patients were more affected by money CS than low $k$ patients which was true for collecting good (Estimate $=0.33, z=6.74$, $P<0.001$ ) and leaving bad shells (Estimate $=-0.31, z=-6.53$, $P<0.001)$. In the model including only controls, high $k$ and low $k$ controls did not differ.

Effect of beverage stimuli in the background ('beverage PIT'). To analyze trials with beverage backgrounds within the full sample, we calculated a model including the fixed factors beverage $(0.5=$ alcohol vs $0.5=$ water $)$, type of instrumental behavior $(0.5=$ collect good shell vs $-0.5=$ leave bad shell) and group ( $0.5=$ patients vs $-0.5=$ controls) as well as the random intercept. We found a significant beverage $\times$ type of instrumental behavior interaction (Estimate $=1.63, z=16.88, P<0.001$ ), indicating that a beverage PIT effect was present in both groups: when collecting good shells, subjects responded more often correctly with water pictures in the background, whereas for leaving bad shells they responded more often correctly with alcohol pictures in the background. The significant beverages $\times$ type of instrumental behavior $\times$ group interaction (Estimate $=-0.58, \quad z=-3.87$, $P<0.001)$ revealed that patients and controls differed in the PIT strength. Calculating separate models for collecting good and leaving bad shells revealed a significant interaction between beverages $\times$ group only in the model for leaving bad shells (Estimate $=0.39, \quad z=3.43, P<0.001$ ), suggesting that patients compared to controls were less able to correctly leave a bad shell when water pictures were presented in the background (Figure 2a, right panel).

Modulation of beverage PIT by choice impulsivity. Including $k$ in the model revealed a significant three-way interaction between beverages $\times$ type of instrumental behavior $\times k$ (Estimate $=-1.54$, $z=6.19, P<0.001)$, indicating that impulsive choice behavior modulated the beverage PIT effect in patients and in controls. Again, this modulation differed between groups as indicated by the significant four-way interaction between beverages $\times$ type of instrumental behavior $\times k \times$ group (Estimate $=0.67, z=2.16$, $P=0.03$ ). We computed separate models to disentangle this interaction. We found no significant main effects or interactions for low $k$ participants (Figure $2 b$, right panel). For high $k$ subjects collecting good shells, we found no significant effects while for leaving bad shells, a significant interaction of beverage $\times$ group revealed that compared to controls, patients were more affected by beverage backgrounds (estimate $=0.48, z=2.83, P=0.005$; see Figure 2c, right panel). When computing models including only patients, we found significant interactions between beverage $\times k$, indicating that high $k$ patients were more affected by beverage backgrounds than low $k$ patients when collecting good shells (Estimate $=-0.80, z=-5.29, P<0.001$ ) and when leaving bad shells (Estimate $=0.89, z=5.96, P<0.001$ ). For controls, we found the same interactions, indicating stronger effects of beverage backgrounds in high- compared to low $k$ controls for collecting good shells (Estimate $=-0.54, z=-2.79, P=0.005$ ) and leaving bad shells (Estimate $=0.47, z=2.64, P=0.008$ ).

\section{DISCUSSION}

We observed stronger PIT effects related to both money CS and beverage stimuli in alcohol-dependent patients compared to controls. In general, positively valenced background stimuli (associated to monetary gains) substantially enhanced accuracy of approach behavior (that is, when collecting good shells) and impaired accuracy when inhibiting approach behavior (that is, when leaving bad shells). Complementary, negatively valenced background stimuli (associated to monetary losses) had the opposite effect on accuracy. Particularly, when patients were confronted with a combination of a negatively valued instrumental cue (for example, a bad shell) and a positively valenced background Pavlovian cue (for example, $+1 €,+2 €$, or water), their behavior was strongly driven by those background cues, rendering them less able than controls to appropriately perform the instrumentally learned inhibition. When accounting for impulsive choice behavior as measured by the delay discounting parameter $k$, we found stronger PIT effects in high impulsive compared to low impulsive participants. Further, the stronger PIT effect in patients compared to controls was only present in the high-impulsive group, whereas low impulsive patients and controls did not differ.

We could successfully replicate our previous finding of stronger PIT effects in alcohol-dependent patients compared to controls in the full sample as well as in a replication sample, for which subjects of previously reported subsamples were excluded. In these previous presented studies, patients were behaviorally more affected by money and by beverage cues, ${ }^{16}$ while the influence of money cues was positively related to stronger activity in the NAcc only in relapsers, but not in abstainers. ${ }^{17}$ The current results extend these data by separately analyzing Pavlovian influences on instrumental approach and avoidance behavior. We showed that patients compared to controls were more susceptible to Pavlovian background cues in general, and particularly to cues associated to monetary gains while they should perform an instrumentally learned inhibition. Therefore, Pavlovian effects may automatically and implicitly bias patients to make decisions that are contrary to their previously formed behavioral intention. Translated to everyday risk situations for relapse, this concept may manifest itself as a strong susceptibility to seduction, which can be a crucial aspect in high-risk situations for relapse.

Surprisingly, we found the same effect of failed behavioral inhibition when pictures of water were presented in the background, while pictures of alcohol decreased instrumentally learned approach. Both effects were more pronounced in patients, particularly in high-impulsive patients. Thus, water pictures exerted appetitive effects similarly to CS paired with winning money, while alcohol stimuli resembled CS paired with losing money. We could further confirm this assumption with data from the forced choice trials: participants preferred water pictures over negative $(-1 €,-2 €)$ and neutral $(0 €)$ money CS as well as water and all money CS over alcohol pictures. When asking subjects to choose between alcohol and water pictures, both patients and controls preferred the water picture, while the aversion against alcohol pictures was even stronger in patients.

The unexpected aversion against alcohol pictures in patients might be related to the long stimulus presentation in our task (for example, $3 \mathrm{~s}$ in each trial during the PIT part). All studies investigating attentional biases towards alcohol-related cues used 
shorter presentation times. For instance, data from a visual probe paradigm shows that alcohol-dependent patients exhibit an attentional bias towards alcohol cues (for example, faster detection of a probe that replaces alcohol cues compared to neutral cues) only when the duration of stimulus presentation is short $(50 \mathrm{~ms})$. When cues were presented for $500 \mathrm{~ms}$, social drinkers showed an increased attentional bias compared to alcohol-dependent patients, and when stimuli were presented for $1250 \mathrm{~ms}$ no attentional bias was found, neither in patients nor in social drinkers. ${ }^{44}$ VollstädtKlein et al. ${ }^{45}$ further showed that patients, who were abstinent for longer than 2 weeks, exhibited the same patterns in attentional orienting towards alcohol cues as healthy controls. These results suggest a vigilance-avoidance pattern towards alcohol-related stimuli that could also explain the stronger dislike of alcohol pictures in our data. Since our stimuli presentation times were relatively long, patients might have successfully applied attentional avoidance- and cognitive evaluation strategies to process those alcohol-related cues, especially in their current status after recent detoxification and within continuous abstinence-oriented treatment. Another possible explanation is that 'general' and 'specific' PIT effects exerted by alcohol pictures might differ in their direction: while we observed a general inhibitory effect of alcohol pictures on instrumental responses to money cues, alcohol pictures might still enhance the approach towards alcohol cues, which we did not test (that is, 'specific' PIT).

In our study, PIT effects were more pronounced in highimpulsive subjects, and above all in high-impulsive patients. Those patients seem to be particularly sensitive to the impact of Pavlovian cues on ongoing behavior. A possible mechanism underlying this overlap between choice impulsivity and susceptibility to Pavlovian cues might be a generally increased sensitivity to rewards. For instance, Hariri et al. ${ }^{46}$ reported the data of an fMRI study showing that higher discounting rates were positively correlated with increased ventral striatal activity in response to both, positive and negative feedback in a Striatal reactivity paradigm. Further, in the context of problem drinking and AUD risk, the combination of steep monetary delay discounting and activity patterns of high ventral striatal and low amygdala reactivity where identified as a possible pathway to increased stress-induced drinking behavior. ${ }^{47}$ In summary, these results imply that impulsive patients with a strong responsiveness to rewards might also be less able to inhibit a behavioral response towards a reward-associated background cue. Accordingly, this higher reward-sensitivity could explain the incentive salience those subjects attribute to Pavlovian cues. Another complementary theoretical approach to explain the susceptibility to Pavlovian cues can be found in the goal- and sign-tracking literature, which describes individual variance in the degree to which Pavlovian cues acquire motivational properties. ${ }^{48,49}$ While goal-tracking individuals tend to approach the position of anticipated reward delivery, sign-trackers excessively approach the position where the stimulus predicting the reward is presented. Related to that construct, Tomie et al. ${ }^{50}$ showed that sign-tracking behavior was associated with higher choice impulsivity: high impulsive compared to low impulsive rats showed remarkably more approach behavior towards the CS. Moreover, sign-tracking as well as impulsive choice behavior further increased after acute administration of ethanol, but only in high impulsive, not in low impulsive animals. In a more recent human study, Garofalo and di Pellegrino ${ }^{51}$ used eye-tracking to identify goal-and sign-tracking behavior during a PIT paradigm. Correspondingly, they found that sign-trackers showed a stronger PIT effect and higher levels of impulsivity compared to goal-trackers. We assume that the strong PIT effects we observed in high-impulsive patients could rely on related mechanisms. Similar to sign-tracking individuals, highimpulsive patients appear to attribute incentive salience to Pavlovian CS, which motivate reward-related actions and thus interfere with instrumentally learned behavior.
We want to address two limitations to be considered when interpreting our data: First, there are a number of sample characteristics that differ between patients and controls, which possibly could have biased our results. For instance, the socioeconomic status of patients was lower compared to controls. Differences in the socioeconomic status might be important for decision-making in the delay discounting task as well as in the PIT paradigm, because it can affect the subjective value of presented money stimuli. ${ }^{52}$ Further, we allowed subjects to smoke before the beginning of the experiment. Since nicotine is known to independently affect reward learning ${ }^{53,54}$ and impulsivity ${ }^{23}$ the current smoking state of subjects might have influenced our results. However, due to the complexity of our statistical models we could not control for differences in socioeconomic status or smoking state. Second, the stronger PIT effects in high-impulsive patients might have simply been explained by their poorer instrumental learning. Indeed, patients and particularly highimpulsive patients achieved lower accuracies than controls during instrumental training (Supplementary Figure 2) and therefore might have responded according to values of the money CS by neglecting instrumental learned stimuli during the PIT phase. To test this assumption, we included participants' achieved accuracy during instrumental training in our model analyzing the PIT phase. Results revealed that instrumental training performance did not affect the stronger PIT effect in patients compared to controls, while this effect remained unchanged when we included impulsivity in the model. Taken together, we conclude that the strong effect of Pavlovian cues on highly impulsive patients' behavior cannot be explained by poorer instrumental training performance.

In conclusion, our findings indicate that during a PIT paradigm, monetary Pavlovian cues as well as pictures of beverages can strikingly influence instrumentally learned behavior. Particularly, high-impulsive alcohol-dependent patients showed inappropriate approach behavior elicited by contextual cues and therefore failed to correctly inhibit previously learned actions. In real life, this trait might render them highly susceptible to seduction by environmental cues that are incongruent with their behavioral intention to stay abstinent. Such cues can be alcohol-related or alcoholunrelated, and both occur in what therapists like to call 'chains of seemingly harmless decisions'.

For example, listening to pleasant music while finding the picture of an old friend may prompt a patient to call that friend, the good food that friend suggests to have at a restaurant might make him accept the friend's invitation, talking about the fun they had with old drinking buddies might make him go see them in a bar next street, and there ultimately be seduced to accept a drink.

Our results bear two implications to help highly impulsive alcoholics abstain despite their strong PIT effect. In the cognitive domain, they could be informed about their susceptibility to context cues and how these can influence everyday behavior in a way that increases relapse risk. On a more implicit, habitual level of behavioral control, these patients might particularly profit from cue-reactivity training, which aims to relearn automatic responses to cues and replaces approach by withdrawal reactions (for example, Eberl et al., ${ }^{55}$ Wiers et al. ${ }^{56}$ ).

\section{CONFLICT OF INTEREST}

The authors declare no conflict of interest.

\section{ACKNOWLEDGMENTS}

We thank the whole team of the LeAD study for fruitful discussions and their relentless work in data acquisition, management and quality control. Supporting Information is available online. This work was funded by the German Research Foundation (Deutsche Forschungsgemeinschaft, DFG FOR 1617 grants: HE 2597/13-1, HE 2597/14-1, HE 2597/15-1, RA 1047/2-1, SM 80/7-1, Zl 1119/3-1, ZI 1119/4-1, WI 
709/10-1, HE 2597/13-2, HE 2597/14-2, HE 2597/15-2, RA 1047/2-2, SCHA 1971/1-2, SM 80/7-2, ZI 1119/3-2, WI 709/10-2) and by the German Federal Minstry of Education and Research (BMBF) grant 01ZX1311H.

\section{REFERENCES}

1 Heinz A, Siessmeier T, Wrase J, Hermann D, Klein S, Grüsser-Sinopoli SM et al. Correlation between dopamine D2 receptors in the ventral striatum and central processing of alcohol cues and craving. Am J Psychiatry 2004; 161: 1783-1789.

2 Drummond DC. What does cue-reactivity have to offer clinical research? Addiction 2000; 95: 129-144.

3 Grüsser SM, Wrase J, Klein S, Hermann D, Smolka MN, Ruf M et al. Cue-induced activation of the striatum and medial prefrontal cortex is associated with subsequent relapse in abstinent alcoholics. Psychopharmacology (Berl) 2004; 175: 296-302.

4 Holmes NM, Marchand AR, Coutureau E. Pavlovian to instrumental transfer: A neurobehavioural perspective. Neurosci Biobehav Rev 2010; 34: 1277-1295.

5 Cartoni E, Moretta T, Puglisi-Allegra S, Cabib S, Baldassarre G. The relationship between specific Pavlovian instrumental transfer and instrumental reward probability. Front Psychol 2015; 6: 1697.

6 Corbit LH, Balleine BW. Double dissociation of basolateral and central amygdala lesions on the general and outcome-specific forms of pavlovian-instrumental transfer. J Neurosci 2005; 25: 962-970.

7 Huys QJM, Cools R, Gölzer M, Friedel E, Heinz A, Dolan RJ et al. Disentangling the Roles of Approach, Activation and Valence in Instrumental and Pavlovian Responding. PLoS Comput Biol 2011; 7: e1002028.

8 LeBlanc KH, Maidment NT, Ostlund SB. Repeated cocaine exposure facilitates the expression of incentive motivation and induces habitual control in rats. PLoS One 2013; 8: e61355.

9 Shiflett MW, Riccie M, DiMatteo R. The effects of amphetamine sensitization on conditioned inhibition during a Pavlovian-instrumental transfer task in rats. Psychopharmacology (Berl) 2013; 230: 137-147.

10 Saddoris MP, Stamatakis A, Carelli RM. Neural correlates of Pavlovian-toinstrumental transfer in the nucleus accumbens shell are selectively potentiated following cocaine self-administration. Eur J Neurosci 2011; 33: 2274-2287.

11 Anderson BA, Folk CL, Garrison R, Rogers L. Mechanisms of habitual approach: Failure to suppress irrelevant responses evoked by previously reward-associated stimuli. J Exp Psychol Gen 2016; 145: 796-805.

12 Anderson BA, Faulkner ML, Rilee JJ, Yantis S, Marvel CL. Attentional Bias for Nondrug Reward is Magnified in Addiction. Exp Clin Psychopharmacol 2013; 6: 499-506.

13 Lovibond PF, Colagiuri B. Facilitation of voluntary goal-directed action by reward cues. Psychol Sci 2013; 24: 2030-2037.

14 Nadler N, Delgado MR, Delamater AR. Pavlovian to instrumental transfer of control in a human learning task. Emotion 2011; 11: 1112-1123.

15 Trick L, Hogarth L, Duka T. Prediction and uncertainty in human Pavlovian to instrumental transfer. J Exp Psychol Learn Mem Cogn 2011; 37: 757-765.

16 Garbusow M, Schad DJ, Sommer C, Jünger E, Sebold M, Friedel E et al. Pavlovianto-instrumental transfer in alcohol dependence: a pilot study. Neuropsychobiology 2014; 70: 111-121.

17 Garbusow M, Schad DJ, Sebold M, Friedel E, Bernhardt N, Koch SP et al. Pavlovianto-instrumental transfer effects in the nucleus accumbens relate to relapse in alcohol dependence. Addict Biol 2015; 21: 719-731.

18 Loree AM, Lundahl LH, Ledgerwood DM. Impulsivity as a predictor of treatment outcome in substance use disorders: Review and synthesis. Drug Alcohol Rev 2015; 34: 119-134.

19 Dougherty DM, Mathias CW, Marsh DM, Jagar AA. Laboratory behavioral measures of impulsivity. Behav Res Methods 2005; 37: 82-90.

20 Heinz AJ, Beck A, Meyer-Lindenberg A, Sterzer P, Heinz A. Cognitive and neurobiological mechanisms of alcohol-related aggression. Nat Rev Neurosci 2011; 12: 400-413.

21 Jentsch JD, Ashenhurst JR, Cervantes MC, Groman SM, James AS, Pennington ZT. Dissecting impulsivity and its relationships to drug addictions. Ann N Y Acad Sci 2014; 1327: 1-26.

22 Dick DM, Smith G, Olausson P, Mitchell SH, Leeman RF, O'Malley SS et al. Review: understanding the construct of impulsivity and its relationship to alcohol use disorders. Addict Biol 2010; 15: 217-226.

23 MacKillop J, Amlung MT, Few LR, Ray LA, Sweet LH, Munafo MR. Delayed reward discounting and addictive behavior: a meta-analysis. Psychopharmacology (Berl) 2011; 216: 305-321.

24 Rupp Cl, Beck JK, Heinz A, Kemmler G, Manz S, Tempel K et al. Impulsivity and alcohol dependence treatment completion: Is there a neurocognitive risk factor at treatment entry? Alcohol Clin Exp Res 2016; 40: 152-160.
25 Hoffman WF, Schwartz DL, Huckans MS, McFarland BH, Meiri G, Stevens AA et al. Cortical activation during delay discounting in abstinent methamphetamine dependent individuals. Psychopharmacology (Berl) 2008; 201: 183.

26 Peters J, Büchel C. Overlapping and distinct neural systems code for subjective value during intertemporal and risky decision making. J Neurosci 2009; 29: 15727-15734.

27 Prévost C, Liljeholm M, Tyszka JM, O'Doherty JP. Neural Correlates of Specific and General Pavlovian-to-Instrumental Transfer within Human Amygdalar Subregions: A High-Resolution fMRI Study. J Neurosci 2012; 32: 8383-8390.

28 Talmi D, Seymour B, Dayan P, Dolan RJ. Human Pavlovian-Instrumental Transfer. J Neurosci 2008; 28: 360-368.

29 Geurts DEM, Huys QJM, den Ouden HEM, Cools R. Aversive Pavlovian Control of Instrumental Behavior in Humans. J Cogn Neurosci 2013; 25: 1428-1441.

30 Stuppäck C, Barnas C, Falk M, Günther V, Hummer M, Oberbauer H et al. Eine modifizierte und ins Deutsche übersetzte Form der Clinical Institute Withdrawal Assessment for Alcohol Scale (CIWA-A). Wien Z Für Suchtforsch 1995; 18: 39-48.

31 Skinner HA, Horn JL. Alcohol Dependence Scale (ADS) user's guide. Addiction Research Foundation: Toronto, Canada, 1984.

32 Wechsler D. WAIS-III: Wechsler Adult Intelligence Scale, Administration and Scoring Manual. Psychological Corporation. Harcourt Brace: San Antonio, Texas, USA, 1997.

33 Mann K, Ackermann K. Die OCDS-G: Psychometrische Kennwerte der deutschen Version der obsessive compulsive drinking scale. Sucht 2000; 46: 90-100.

34 Lehrl S. Mehrfachwahl-Wortschatz-Intelligenztest: MWT-B. Spitta: Balingen, Germany, 2005.

35 Schmidt LG, Gastpar M, Falkai P, Gaebel W. Evidenzbasierte Suchtmedizin. Behandlungsleitlinie Substanzbezogener Störungen. Deutscher Ärzteverlag: Köln, Deutschland, 2006.

36 Bjelland I, Dahl AA, Haug TT, Neckelmann D. The validity of the Hospital Anxiety and Depression Scale: an updated literature review. J Psychosom Res 2002; 52: 69-77.

37 Jacobi F, Mack S, Gerschler A, Scholl L, Höfler M, Siegert J et al. The design and methods of the mental health module in the German Health Interview and Examination Survey for Adults (DEGS1-MH). Int J Methods Psychiatr Res 2013; 22: 83-99.

38 Wittchen HU, Pfister H. DIA-X-Interviews: Manual für Screening-Verfahren und Interview, Swets Test Services: Frankfurt, Germany, 1997.

39 Pooseh S, Bernhardt N, Guevara A, Huys QJ, Smolka MN. Value-based decisionmaking battery: A Bayesian adaptive approach to assess impulsive and risky behavior. Behav Res Methods 2017; 1-14.

40 Mazur JE. An adjusting procedure for studying delayed reinforcement. In M.L. Commons, J. E. Mazur, J. A. Nevin \& H. Rachlin. Quantitative analysis of behavior: The effect of delay and intervening events on reinforcement value; 5 : 55-73. Erlbaum: Hillsdale, Michigan, USA, 1987.

41 Brainard DH. The psychophysics toolbox. Spat Vis 1997; 10: 433-436.

42 Pelli DG. The VideoToolbox software for visual psychophysics: Transforming numbers into movies. Spat Vis 1997; 10: 437-442.

43 R Development Core Team (2008). R: A language and environment for statistical computing. R Foundation for Statistical Computing, Vienna, Austria. http://www. R-project.org.

44 Noël X, Colmant M, Van Der Linden M, Bechara A, Bullens Q, Hanak C et al. Time course of attention for alcohol cues in abstinent alcoholic patients: the role of initial orienting. Alcohol Clin Exp Res 2006; 30: 1871-1877.

45 Vollstädt-Klein S, Loeber S, Von der Goltz C, Mann K, Kiefer F. Avoidance of alcohol-related stimuli increases during the early stage of abstinence in alcoholdependent patients. Alcohol Alcohol 2009; 44: 458-463.

46 Hariri AR, Brown SM, Williamson DE, Flory JD, de Wit H, Manuck SB. Preference for immediate over delayed rewards is associated with magnitude of ventral striatal activity. J Neurosci 2006; 26: 13213-13217.

47 Nikolova YS, Knodt AR, Radtke SR, Hariri AR. Divergent responses of the amygdala and ventral striatum predict stress-related problem drinking in young adults: possible differential markers of affective and impulsive pathways of risk for alcohol use disorder. Mol Psychiatry 2016; 21: 348.

48 Flagel SB, Akil H, Robinson TE. Individual differences in the attribution of incentive salience to reward-related cues: Implications for addiction. Neuropharmacology 2009; 56: 139-148.

49 Robinson TE, Flagel SB. Dissociating the predictive and incentive motivational properties of reward-related cues through the study of individual differences. Biol Psychiatry 2009; 65: 869-873.

50 Tomie A, Grimes KL, Pohorecky LA. Behavioral characteristics and neurobiological substrates shared by Pavlovian sign-tracking and drug abuse. Brain Res Rev 2008; 58: 121-135.

51 Garofalo S, di Pellegrino G. Individual differences in the influence of taskirrelevant Pavlovian cues on human behavior. Front Behav Neurosci 2015; 9: 163.

52 Green L, Myerson J, Lichtman D, Rosen S, Fry A. Temporal discounting in choice between delayed rewards: the role of age and income. Psychol Aging 1996; 11: 79.

53 Olausson P, Jentsch JD, Taylor JR. Nicotine enhances responding with conditioned reinforcement. Psychopharmacology (Berl) 2004; 171: 173-178. 
54 Hogarth L, Chase HW. Evaluating psychological markers for human nicotine dependence: Tobacco choice, extinction, and Pavlovian-to-instrumental transfer. Exp Clin Psychopharmacol 2012; 20: 213.

55 Eberl C, Wiers RW, Pawelczack S, Rinck M, Becker ES, Lindenmeyer J. Approach bias modification in alcohol dependence: do clinical effects replicate and for whom does it work best? Dev Cogn Neurosci 2013; 4: 38-51.

56 Wiers CE, Stelzel C, Park SQ, Gawron CK, Ludwig VU, Gutwinski S et al. Neural correlates of alcohol-approach bias in alcohol addiction: the spirit is willing but the flesh is weak for spirits. Neuropsychopharmacology 2014; 39: 688 .
This work is licensed under a Creative Commons AttributionNonCommercial-NoDerivs 4.0 International License. The images or other third party material in this article are included in the article's Creative Commons license, unless indicated otherwise in the credit line; if the material is not included under the Creative Commons license, users will need to obtain permission from the license holder to reproduce the material. To view a copy of this license, visit http:// creativecommons.org/licenses/by-nc-nd/4.0/

(c) The Author(s) 2017

Supplementary Information accompanies the paper on the Translational Psychiatry website (http://www.nature.com/tp) 